\title{
LXXXVIII. Critical electron velocities for the production of luminosity in atmospheric neon
}

\section{Frank Horton Sc.D. \& Ann Catherine Davies M.Sc.}

To cite this article: Frank Horton Sc.D. \& Ann Catherine Davies M.Sc. (1921) LXXXVIII. Critical electron velocities for the production of luminosity in atmospheric neon , Philosophical Magazine Series 6, 41:246, 921-940, DOI: 10.1080/14786442108636287

To link to this article: http://dx.doi.org/10.1080/14786442108636287

曲 Published online: 08 Apr 2009.

Submit your article to this journal $\lceil\pi$

Џ Article views: 2

Q View related articles $₫$ 
2. The hypothesis of the orientation of the elementary crystals seems to be the most probable. The appearance of the effect in sputtered plates cannot be argued against this hypothesis, since the sputtered particles are undoubtedly of more than molecular dimensions.

The fact that no increase of photo-electric current appears until a certain minimum current is reached, indicates that a certain minimum field is necessary to orient an elementary crystal, the further increases being due to the larger numbers. so oriented.

Further, the fact that a second series of readings with a plate gives the irregular results described previously, would seem to indicate that the structure of the metal had been in some way weakened. The greater rises under these conditions would likewise support the view.

The validity of the hypothesis could, however, be best tested by working with plates cut perpendicular to the crystal axis of a large bismuth crystal. $\Lambda$ further test might be obtained from the measurement of change of contact difference of potential under the conditions existing in this experiment.

Palmer Physical Laboratory,

Princeton, N.J.

LXXXVIII. Critical Electron Velocities for the Production of Luminosity in Atmospheric Neon. By Frank HorTon, Sc.D., Professor of Physics in the University of London, and Ann Catherine Davies, M.Se., Assistant Lecturer in Physics in the Royal Holloway College, Englefield Green *.

$\mathbf{I}^{\mathrm{N}}$

$N$ a recent paper $\dagger$ the authors have shown that in atmospheric neon there are two critical electron velocities associated with the production of radiation from the gas, and three critical electron velocities associated with the production of ionization, the values of these velocities being :

Minimum radiation velocity . . 11.8 volts.

Second radiation velocity . . . 17.8,

Minimum ionization velocity . . 16.7",

Second ionization velocity . . 20.0,

Third ionization velocity . . . . 22.8,

In view of the fact that neon is the only gas for which more than one critical ionization velocity has been found under conditions which preclude the possibility of the

* Communicated by the Authors.

+ Proc. Roy. Soc. A. vol. xcviii. p. 124 (1920). 


\section{Prof. Horton and Miss Davies on Electron Velocities}

higher critical velocities being concerned with the ejection of a second electron from an alrealy ionized atom, it seems desirable to mention briefly the precautions taken to ensure the purity of the gas used in these experiments. The neon was puritied by Dr. Aston of Cambridge, using the elaborate fractionation apparatus which he devised for this purpose. The process of purification was accompanied by frequent determinations of the density of the gas, and was continued long beyond the stage when no alteration of density could be detected. In admitting the gas to the apparatus adequate precautions were taken to remove any impurities which might have found their way into the neon from the glass walls of the vessel in whicli it was stored, or from the walls of the tubes through which it passed, and, during most of the experiments, the pure gas was slowly streaming through the ionizationchamber. With these precautions the only possible impurity in the gas used was helium, and in view of the lengthy and careful treatment of Dr. Aston it is unlikely that more than a minute trace of helium was present. A spectroscopic examination of the gas showed no lines but those of the neon spectrum. The presence of helium in sufficient quantity might have accounted for the detection of a critical ionization velocity at 20 volts-approximately the resonance velocity for electrons in helium,--but the experiments about to be described show that this electron velocity is essential for the production of the principal series lines in the neon spectrum.

In view of the results of the positive ray experiments of Sir J. J. Thomson * and of the more recent experiments of Dr. Aston $\dagger$, which have shown that atmospheric neon contains two constituents of atomic weights 20.00 and 22.00 and possibly a small proportion of a third constituent of atomic weight 21 , the detection of three critical electron velocities for ionization of the gas is of particular interest. In order to explain his results, Dr. Aston assumed that the constituents of atmospheric neon are isotopes, an assumption which is justified by the fact that according to Moseley's theory of atomic numbers there can be no unknown element of atomic weight between 20 and 23 . The existence of three isotopes would not, however, be expected to lead to three different ionization velocities, since the atoms of: isotopes have the same nuclear charge and the same number of surrounding electrons, which would presumably be dis-

* J. J. 'Thomson. ' Rays of Positive Electricity' p. 112 (1913).

$\dagger$ F. W. Aston, Phil. ITag. vol. xxxix, p. 449 (19:0). 
for Production of Luminosity in Atmospheric Neon. 923

tributed in the same way about the nucleus in each case. The existence of the three ionization velocities would be expected if atmospheric neon consisted of three different elements, and in this case it would be possible to excite the spectrum of each element in turn by gradually increasing the speed of the stream of electrons bombarding the gas atoms. It was with the view of obtaining some further information about the three ionizing velocities that the experiments described in the present paper were undertaken. Some preliminary experiments, referred to in our earlier paper, had shown that in order to obtain conclusive evidence it wonld be necessary to use some arrangement for concentrating the rather fueble glow which appeared in the gas at certain velocities of the electron stream. This concentration of the luminosity was obtained by using a stronger magnetic field in the manner described below.

\section{Description of Apparatus*.}

Fig. 1 .

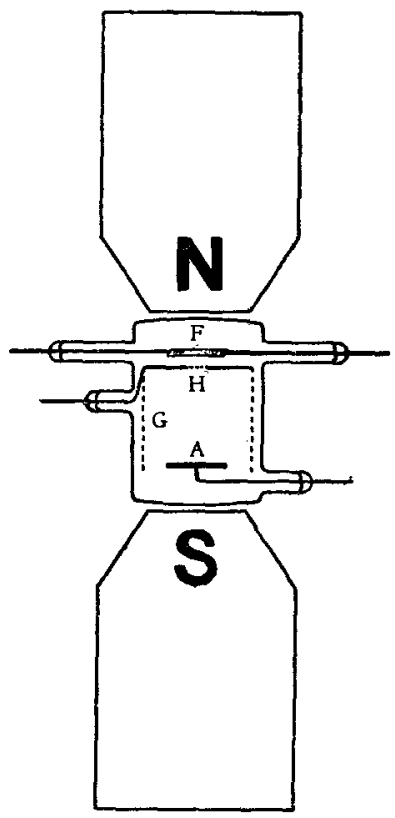

The source of electrons was a platinum filament F (fig. 1) covered with a thin coating of lime. This was about $8 \mathrm{~mm}$.

*. I wish to acknowledge my indebtedness to the Government Grant Committee of the Fioyal Society for the means of purchasing some of the apparatus and materials used in this research.-F. H. 


\section{Prof. Horton and Miss Davies on Electron Velocities}

long and $1 \mathrm{~mm}$. wide, and was situated horizontally and with its middle over the hole $\mathrm{H}(1.5 \mathrm{~mm}$. in diameter $)$ in the centre of a platinum disk which nearly filled the crosssection of the discharge chamber and served to screen off the light of the glowing filament from the lower part of the tube. Round the edges of the platinum disk was fixed a cylindrical piece of fine platinum gauze $G$, about $1.5 \mathrm{~cm}$. long. The hole $\mathrm{H}$ was also covered with a small piece of similar fine gauze so as to improve the electric screening of the space below $\mathrm{H}$. 'The anode $\mathrm{A}$ was a circular platinum plate, $1 \mathrm{~cm}$. in diameter, fixed horizontally just above the level of the open end of the gauze as indicated in the figure. The total length of the ionization chamber was about $2.5 \mathrm{~cm}$., so that it could be easily arranged between the pole pieces $\mathrm{N}$ and $S$ of an electromagnet, which, by producing a strong magnetic field along the axis of the tube, concentrated the stream of electrons passing through $\mathrm{H}$ into a parallel beam. When the velocity of the electron stream was raised to a suitable value, the luminosity thus concentrated along the axis of the tube was very much brighter than the diffused ylow which filled most of the space when no magnetic field was used. The spectrum of the luminosity was viewed by means of a Hilger wave-length spectroscope, the slit of which was arranged so as to be illuminated by the light produced along the axis of the tube between the grid and anode. It was screened by the platinum disk from the direct light of the glowing filament. All the platinum electrodes were boiled for several days in strong nitric acid before being fitted into the apparatus, and the usual precautions were taken for ridding the glass walls of occluded gases.

In the diagram the connexions to the pump \&c. are not shown. The arrangements for circulating pure neon through the apparatus during the observations were similar to those described in the paper already referred to. 'The gas passed from the storage bulb down a fine capillary tube to the discharge chamber, which it entered from a U-tube containing a little carbon and immersed in liquid air. Another tube connected the apparatus to the pumping system.

In some of the experiments which were made with this apparatus, in addition to observing the spectrum of the luminosity produced in the gas under different conditions, the currents between the eloctrodes were measured at the same time in order to ascertain whether sudden changes in the spectrum of the gas were connected with changes in the gas ionization. The currents were measured by means of a 
sensitive moving-coil galvanometer which was situated in a room adjoining that in wiich the neon spectrum was under observation, the latter room being kept in darkness for the letter detection of faint lines.

\section{Experimental Results.}

For the purpose of determining the limiting electron velocities required to produce particular lines in the neon spectrum, it was necessary that the bombarding electrons should suffer no change of velocity throughout the space which was viewed by the slit of the spectroscope, except such change as results from collisions with gas atomis. To secure this condition, the grid and anode were maintained at the same potential by connecting each to the positive terminal of an insulated battery, the negative end of which was connected to the negative end of the filament. The maximum velocity of any of the electrons passing through the hole $\Pi$ is thus the sum of that due to the applied potential difference and the velocity of emission of the electrons from the filament. If the maximum velocity of emission is determined, the velucity of the swiftest electrons passing through $\mathrm{H}$ under any applied potential difference is known. The maximum velocity of the electrons passing through $H$, when any given line is first seen in the spectroscope, thus gives an upper limit for the critical electron velocity required for the excitation of the particular line in question. The velocity of emission was determined by finding what potential difference it was necessary to apply between the filament and the grid in order to prevent any electrons from the filament reaching the grid. In taking this maximum velocity as the critical velocitv, it is assumed that the electrons having this velocity are sufficiently numerous to produce the given line with such intensity as to be visible. In our earlier paper a method was describ d by means of which the velocity of the electrons actually prodncing the radiation or ionization current under observation could be obtained. With the present apparatus it was not possible to determine the actual electron velocity in a similar manner; and the electron velocities stated as used in the present research are in all cases the maximum velocities in the electron stream under the conditions of experiment.

The first appearance of luminosity and the alterations in its spectrum were carefully observed as the velocity of the electron stream was gradually raised from about 10 volts to Phil. Mag. S. 6. Vol. 41. No. 246. June 1921. $3 \mathrm{P}$ 


\section{Prof. Horton and Miss Davies on Electron Velocities}

30 or 40 volts. These observations were repeated with different pressures of neon in the apparatus-up to about $1.5 \mathrm{~mm}$., - and at each pressure with various intensities of the electron stream. With the filament at a high temperature and with about 40 volts potential difference between the filament and the grid, the spectrum of the glowing gas contained the lines given in the following table. The relative intensities of the lines are also given, together with the series to which they belong according to the recent classification of the lines of the neon spectrum by Paschen *.

\section{TABLe I.}

Lines observed in the spectrum of the glow when the electron velocity was about 40 volts.

\begin{tabular}{|c|c|c|c|c|c|}
\hline$\lambda$. & Intensity. & Series. & $\lambda$. & Intensity & Series \\
\hline 6717 & 0.5 & $1 \cdot 5, s_{2} \quad 2, p_{5}$ & 5764 & 1 & $2, p_{0}-4, d_{4}^{\prime}$ \\
\hline 6678 & $0 \%$ & $1 \cdot 5, s_{2}-2, p_{4}$ & 5748 & 0.5 & $2, p_{9}-4, d_{1}^{\prime}$ \\
\hline 6599 & 0.5 & $1 \cdot 5, s_{2}-2, p_{2}$ & 5690 & $0 \cdot 5$ & $2, p_{10}-3 \cdot 5, s_{3}$ \\
\hline 533 & 0.5 & $1 \cdot 5, s_{3}-2, p_{7}$ & 5657 & 0.5 & $2, p_{7}-3 \cdot 5, s_{1}^{\prime \prime \prime \prime}$ \\
\hline 507 & 2 & $1 \cdot 5, s_{4}-2 p_{8}$ & 5563 & 0.5 & $2, p_{8}-3.5, s_{1}^{\prime \prime \prime}$ \\
\hline 6402 & 8 & $1 \cdot 5, s_{5}-2, p_{9}$ & 5401 & 3 & $1 \cdot 5, s_{4}-2, p_{2}$ \\
\hline 6383 & 1 & $1 \cdot 5, s_{4}-2, p_{7}$ & 5341 & 3 & $2, p_{10}-4, d_{5}^{2}$ \\
\hline 305 & 0.5 & $1 \cdot 5, s_{4}-2, p_{6}$ & 5331 & 3 & $2, p_{10}-4, d_{3}$ \\
\hline 266 & 2 & $1 \cdot 5, s_{3}-2, p_{5}$ & 5298 & 0.5 & $2, p_{1}-4 \cdot 5, s_{2}$ \\
\hline 217 & 0.5 & $1 \cdot 5, s_{5}-2, p_{\tau}$ & \multicolumn{3}{|c|}{ Sone faint lines. } \\
\hline 164 & 1 & $1 \cdot 5, s_{3}-2, p_{2}$ & 5222 & 1 & $2, p_{8}-4 \cdot 5, s_{4}$ \\
\hline 143 & 8 & $1.5, s_{5}-2, p_{6}$ & 5204 & 1 & $2, p_{6}-5, d_{1}{ }^{2}$ \\
\hline 096 & 4 & $1.5, s_{4}-2, p_{4}$ & 5189 & 2 & $2, p_{9}-4 \cdot 5, s_{5}$ \\
\hline 74 & 4 & $1 \cdot 5, s_{4}-2, p_{3}$ & \multicolumn{3}{|c|}{ Some blurred } \\
\hline 6030 & 1 & $1 \cdot 5, s_{4}-2, p_{2}$ & 5145 & 2 & $2, p_{4}-4 \cdot 5, s_{1}^{\prime \prime \prime}$ \\
\hline 5976 & 1 & $1 \cdot 5, s_{5}-2, p_{5}$ & 5116 & 2 & $2, p_{10}-3$ \\
\hline 5945 & 3 & $1.5, s_{5}-2, p_{4}$ & 5080 & 2 & $2, p_{8}-5, d_{4}$ \\
\hline $\begin{array}{l}852 \\
820\end{array}$ & 10 & J $5, s_{2}-2, p_{1}$ & 5038 & 3 & $2, p_{9}-5, d_{i}^{\prime}$ \\
\hline & 00 & $2, p_{8}$ & & 1 & \\
\hline
\end{tabular}

It was found that the minimum electron velocity at which a glow ever appeared in the gas was 20 volts. Over the range of pressures investigated, the point at which the glow appeared was independent of the pressure of neon in the apparatus provided this was not too low (above about $0.05 \mathrm{~mm}$.), but it was found to depend somewhat upon the intensity of the elestron stream, and also upon that of the magnetic field used to concentrate the luminosity. In general, as the speed of the electron stream was gradually raised, the brightest lines in the spectrum of the luminosity

* F. Paschen, Ann. der Physik, vol. lx. p. 405 (1919). 
first produced in the gas were those given in the table below.

\section{TABLe II.}

The brightest lines in the spectrum of the luminosity first produced.

$\lambda$.

$6+02$

6143

6096

6074

59.15

5852

5401
Series.

$1 \cdot 5, s_{5}-2, p_{9}$

$1 \cdot 5, s_{5}-2, p_{6}$

$1 \cdot 5, s_{4}-2, p_{4}$

$1 \cdot 5, s_{4}-2, p_{3}$

$15, s_{\overline{3}}-2, p_{1}$

$1 \cdot 5, s_{2}-2, p_{1}$

$1 \cdot 5, s_{4}-2, p_{1}$

With suitable values of the intensity of the electron stream and gas pressure, slight differences in the minimum voltages at which these bright lines first became visible were detected. In these cases the line $\lambda 5852$ was tho first to appear, and then the lines of higher wave-length in the table, and finally $\lambda 5401$; but the minimum electron velocities at which $\lambda 5852$ and $\lambda 5401$ were ever observed did not differ by more than 0.3 volt. With an intense electron stream, all the lines in Table $I$. of longer wavelength than $\lambda 5852$, and also the line $\lambda 5401$, could be identified in the spectrum of the glow a few tenths of a volt after it was first produced, and it was found that the visibility of these lines of higher wave-length than $\lambda 5852$ depended solely upon the general brightness of the luminosity produced in the gas. From Table I. it may be seen that all these lines have been classified by Paschen as belonging to sequences of the type $(1 \cdot 5, s-m, p)$; i. e., they are principal series lines. It was therefore concluded that the spectrum of the glow produced at 20 volts contained all the principal series lines of Table I., the slight differences in the minimum electron velocities at which different lines were first observed being due entirely to the relative intensities of the different lines.

As the speed of the electrons was further raised about 2 volts or more, certain lines in the blue, green, and yellow, of which those given in Table III. were the brightest suddenly made their appearance. 
TABLe III.

The brightest lines of those which suddenly appeared in the spectruin of the glow at about 23 volts.

$\lambda$.

5764

5341

5331

5189

5145

5116

5080

5038
Series.

$2, p_{9}-4, d_{4}^{\prime}$

2, $p_{10}-4, d_{5}$

$2, p_{10}-4, d_{3}$

$2, p_{\mathrm{g}}-4 \cdot 5, s_{\mathrm{b}}$

$2, p_{4}-4 \cdot 5, s_{1}^{\prime \prime \prime}$

2. $p_{10}-3 \cdot 5, s_{1}^{\prime \prime}$

$2, p_{8}-5, d_{4}$

$2, p_{3}-5, d_{4}^{\prime}$

These lines, according to Paschen's classification, all belong to sequences of the type $(2, p-m, s)$ or $(2, p-m, d)$ and are subordinate series lines. A few tenths of a volt after this second set of lines became visible, some of them were distinctly brighter than other lines which were visible at a lower electron velocity and which had gradually brightened as the potential difference was raised. The appearance of the two sets of lines at different electron velocities is therefore not merely due to their production with very different intensities at some definite minimum electron velocity, the same for both, but is due to a genuine difference in the energy required to excite the two sets. Further evidence in support of this view was obtained from a series of measurements of the ionization in the space between the grid and anode taken with the galvanometer while the spectrum was being examined. In obtaining these measurements, a potential difference was maintained between the grid and anode opposite in direction to that between the filament and the grid, and exceeding the latter potential difference by a constant amount of 3 volts, so that none of the electrons from the filament could reach the anode. During the observations the potential difference accelerating the electron stream from the filament was measured by means of a very high resistance voll meter connected between the grid and the negative end of the filament. In the curve A of fig. 2, which represents the results obtained with gradually increasing electron velocity, it may be observed that there is a sudden large increase of current at $22 \cdot 2$ volt.. The lines $\lambda 5852$ and $\lambda 5401$ and some of the brighter red lines were visible before this increase of current occurred, and the rather feeble glow visible in the tube before $22 \cdot 2$ volts brightened euormously when the current increase occurred. 
for Production of Luminosity in Atmospheric Neon. 929

This increase of luminosity was accompanied by the appearance of the remaining red and orange lines of Table I., which had previously been of too small an intensity to be visible; but the lines of Table III. and, in fact, all the lines belonging to subordinate series were absent. The curve shows that alter the large increase of ionization, probibly caused by a sudden nentralization of the space charge near the filament, the current increased steadily with increasing electron velocity until 23.4 volts, after which it began to increase at a more rapid rate. At the stage where this more rapid increase

Fig. 2.

Curve A.

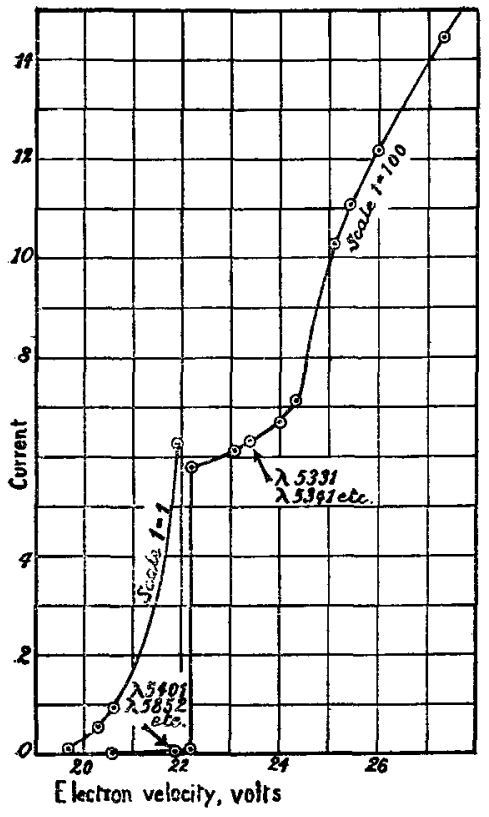

Curve B.

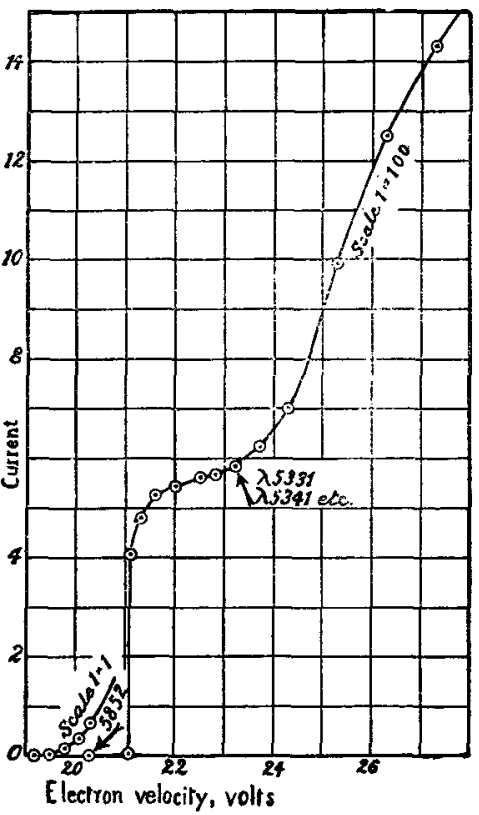

began the brighter green lines given in Table III. suddenly flashed out, and as the electron velocity was still further increased the remaining subordinate series lines of Table I. gradually made their appearance. 'The curve B of fig. 2 gives the values of the ionization current corresponding to different values of the electron velocity as this was gradually reduced from about 27 volts. At 23.2 volts, in this curve, the last remaining subordinate series lines suddenly disappeared, the other's having gradually faded out earlier as the electron velocity was reduced. After this point the current decreased less rapidly as the velocity was reduced until $21 \cdot 1$ volts, when 
a sudden large drop in the magnitude of the current occurred. The brighter principal series lines were however still visible, and these did not all disappear until the electron velocity was 20.3 volts, when the yellow line $\lambda 5852$ vanished. With an electron velocity of 24 volts the green lines $\lambda 5331$ and $\lambda 5341$ which are two of the brightest of the subordinate series lines, were as bright as the principal series green line $\lambda$ 5401. From curve $A$ it may be seen that $\lambda 5401$ was visible when the current measured in arbitrary units was 6.28 , whereas $\lambda 5331$ and $\lambda 5341$ were still not visible when the current measured in the same units was 600 . The fact that $\lambda 5331$ and $\lambda 5341$ and the other subordinate series lines require a higher electron velocity for their production than do $\lambda 5401$ and the other principal series lines, is thus clearly not due to a mere difference in the relative intensities of the two sets of lines. The results of many series of observations of the changes in the spectrum with increase of the electron velocity seemed to indicate that a minimum velocity of about 23 volts was required to produce the subordinate series lines.

The set of lines which appeared at the higher electron velocity contained, in addition to the brighter lines (given in Table IIJ.), all the other subordinate series lines in Table I. These made their appearance a small fraction of a volt after the brighter lines were visible, when the general brightness of the luminosity was high. Thus, although the principal series lines are produced at 20 volts and the subordinate series lines at 23 volts, the fainter members of these series in each case require for visibility a greater concentration of radiant atoms than exists when only the swiftest members of the electron stream have the necessary minimum velocity.

From the results of these experiments, and from our previously determined values of the ionization velocities for electrons in neon, it seemed probable that the appearance of luminosity in the gas was connected with the 20.0 volt ionization, but that the production of the complete neon spectrum required the third type of ionization, found in our earlier work to begin at $22 \cdot 8$ volts. Some confirmation of this view is provided in the curves already given in fig. 2 . In both of these curves the limiting electron velocity for the visibility of any subordinate series lines is practically coincident with the beginning of an alteration in the rate of change of the ionization current with change of electron velocity, such as is usually taken to indicate a change in the type of ionization occurring. Moreover, the bend in the curve occurs at about 23 volts, the velocity at which the third type of ionization begins. In order to test further our view regarding the connexion between the ionization velocities 
for Production of Luminosity in Atmospheric Neon. 931 20.0 volts and 22.8 volts, and the excitation of the lines of the principal and subordinate series respectively, many series of observations of the currents between the different electrodes were made at the same time as observations of the spectrum of the glow when the electron velocity was varied. Examples of some of the curves obtained, with the points at which representatives of the two groups of lines first appeared, are given in figs. 3 and 4.

The series of observations represented in fig. 3 was taken with the grid and anode connected together and to one terminal of the galvanometer, the other galvanometer terminal being connected through the battery to the negative end of Fig. 3.

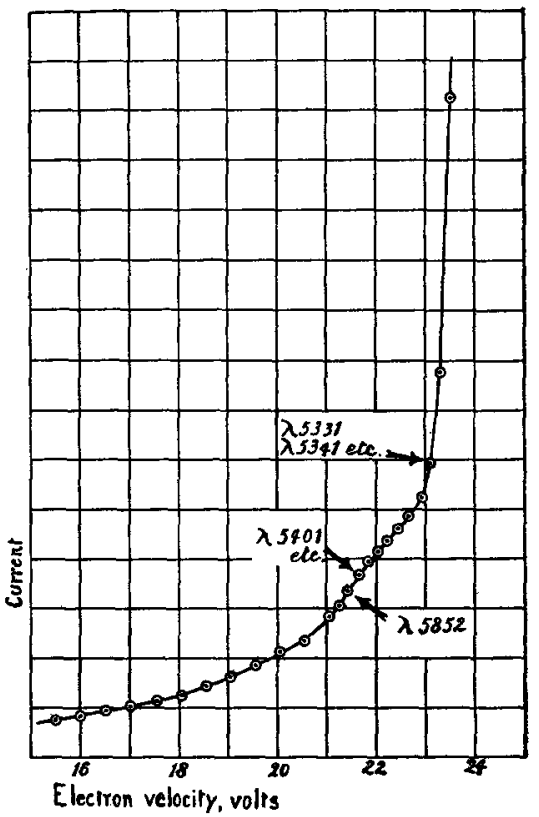

the filament. Thus the electrons, after being accelerated up to the grid by the applied potential difference, passed into a field-free space and, when the ionization velocity wis reached, were able. to produce ionization over a considerable distance. Hence, with this arrangement, the luminosity when it first appeared extended over a much bigger length of the distance between the grid and anode than was the case when the electrons were retarded between these electrodes. The current measured by the galvanometer is, with this arrangement, the total current passing between the filament and the other two electrodes. The curve of fig. 3 shows three bends: 
the first at about 17 volts indicating the beginning of ionization, the second at 20.5 volts indicating an increased production of ionization, and the third and most marked bend at 23.0 volts denoting a still further increased production of ionization. The first appearance of luminosity occurred between 21.2 volts and 21.4 volts, the yellow line $\lambda 5852$ being then visible in the spectroscope. At $21 \cdot 65$ volts, $\lambda 5401$ and the other lines of Table II. were discernible. At an electron velocity of $23 \cdot 1$ volts the lines $\lambda 5341, \lambda 5331$, and $\lambda 5031$ could be seen faintly; and at an electron velocity

Fig. 4.

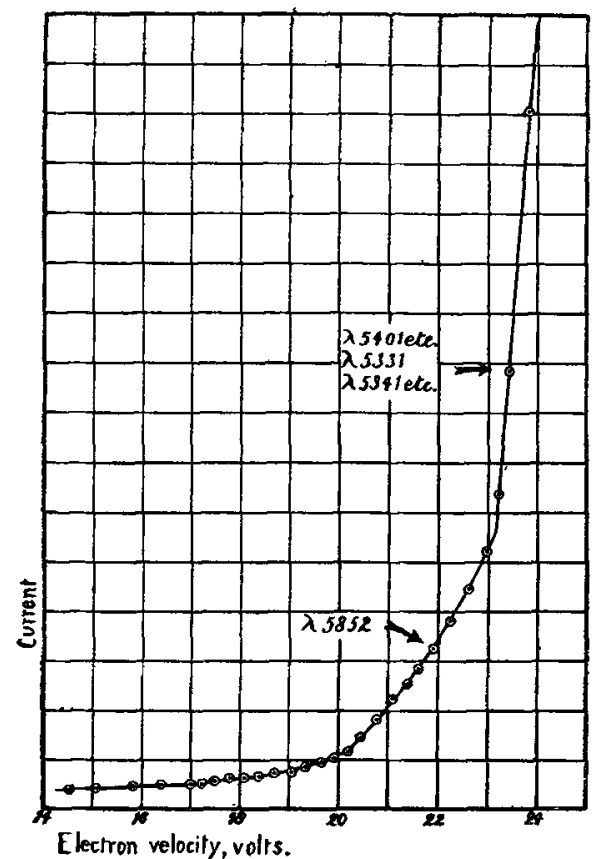

of 23.5 volts all the lines of Table III. could be seen, and the whole spectrum was much brighter. For this series of observations a fairly strong magnetic field was used for the concentration of the Juminosity.

In fig. 4 a series of observations of the ionization current and spectrum variations with alteration of the electron velocity, in the absence of a magnetic field, is represented. The arrangement of electric fields was very similar to that used in obtaining the curve of fig. 3. As was to be expected, 
for Production of Luminosity in Atmospheric Neon. 933

the luminosity when the magnetic field was noton, extended thronghout the whole of the space between the grid and anode instead of being concentrated into a brighi central column. The lines seen through the spectroscope were very much less intense in the absence of the maynetic field, and in some cases, even though the luminosity could be seen faintly at electron relocities between 20 volts and 21 volts on observing the apparatus directly, no line but $\lambda 5852$ could be seen through the spectruscope until the ionization velocity 22.8 rolts was exceeded and the second group of lines appeared; the remaining lines of Table II. then became visible also. This was the case in the series of observations represented in fig. 4. The curve shows ionization beginning at about 17 volts, an increased rate of production of ionization beginning at $20 \cdot 2$ rolts and another such increase at $23 \cdot 1$ volts. At $20 \cdot 8$ volts a faint luminosity was observed in the tube, and the yellow line $\lambda 5852$ was seen through the spectroseope at $21 \cdot 9$ volts. No other lines appeared until 23.4 volts, at which relocity all the brighter green and red lines of Tables II. and III. could be seen faintly. After the completion of these observations, the spectrum was observed with the magnetic field on and with an electron velocity of $22 \cdot 5$ volts, $i$. e. with the relocity just below that at which the third type of ionization hegins. All the brighter principal series lines, including the green line $\lambda 5401$, were distinctly visible and were moch brighter than they had previously been at 24 volts; but none of the subordinate series lines were present. From these and other similar curves it was concluded that a minimum electron velocity of $22 \cdot 8$ volts is requires for the production of the subordinate series lines, but that the principal serie; lines can be stimulated by electrons having a smaller velocity than this.

The fact that the lines of 'Table II. are generally first observed at some velocity after the 20.0 volts ionization has been detected, but before the 22.8 rolts ionization occurs, combined with the fact that no luminosity was ever observed below $20 \cdot 0$ volts, constitutes fairly conclusive evidence that the stimulation of these principal series lines results from the occurrence of the 20.0 volts type of ionization and not from the 16.7 rolts type of ionization. Further evidence on this point was obtained from sereral series of simultaneous observations of the ionization current and the spectrum of the luminosity laken with different intensities of electron stream and gas pressure. In one of these series the value of the ionization current when there 


\section{Prof. Horton and Miss Davies on Electron Velocities}

was the first appearance of luminosity was $8 \cdot 1$ arbitrary units, the electron velocity being then slightly greater than 20 volts. In another series of observations, in which the gas pressure was higher and in which a more intense electron stream was used, the ionization current at $19 \cdot 7$ volts was as great as 79 of the same units without there being any trace of luminosity, though as soon as the electron velocity exceeded 20.0 volts a glow appeared in the gas. In neither of these series of observations was a magnetic field used. Since at 20 volts the number of radiating centres is sufficient for luminosity with an ionization current of 8.1 units, the absence of visible light at $19 \cdot 7$ volts with an ionizaticn current of 79 units must be due to the electron velocity being too low, rather than to the glow being produced too faintly to be visible.

The experiments have therefore shown that the lines which we have observed in the neon spectrum make their appearance in two stages, as the relocity of the exciting electron stream is raised. The lines of the principal series type depend for their production upon the presence of the $20^{\circ} 0$ volts ionization, while the subordinate series lines require the higher ionization velocity of 22.8 volts. No lines in the visible spectrum result. from the ionization which occurs at 16.7 volts. With the exception of the green line $\lambda 5401$, all the lines produced by the 20.0 volts ionization lie in the red and orange parts of the spectrum, while the lines which require $22 \cdot 8$ volts for their excitation are in the green and blue parts of the spectrum.

The difference in the velocities needed to excite these two sets of lines is beautifully illustrated if the experiments are carried out at a pressure rather higher than those used in most of the foregoing experiments. With a pressure of about $1 \mathrm{~mm}$., and with the inagnetic field applied, a brilliant pencil of light can be obtained along the axis of the discharge tube. If the electric fields are arranged so that the electrons are accelerated from the filament to tie grid and then retarded between the grid and the anode by an opposing tield sufficiently strong to prevent any of them from reaching the latter electrode, luminosity appears when the velocity of the stream reaches 20 volts ; it is then of a blood-red colour, and is confined to a thin layer of gas just under the grid. As the accelerating potential difference is slowly raised, this luminosity gradually extends downwards towards the anode, and, after 23 volts has been passed, the lower end, only, of the column is red, while the rest of it is a brilliant orange colour. 
for Production of Luminosity in Atmospheric Neon. 935

In the red part of the glow none but principal series lines are being produced, and, since the velocity of the electrons from the filament gradually decreases as they travel from the grid towards the anode, the experiment clearly shows that these lines can be stimulated when the relocity of the bombarding electrons has been reduced to a value too small to excite the complete neon spectrum.

An appearance which is the reverse of that just described may be obtained by arranging the electric fields so that they both tend to accelerate the electron strea $m$ towards the anode, but with the difference of potenijal between the filament and the grid considerably less tban that necessary for ionization by collisions to take place just below the grid. For instance, in one such experiment, at a pressure of about $1.4 \mathrm{~mm}$., the potential difference between the filament and the grid was maintained at 8 volts and the potential difference between the grid and anode was gradually raised. No luminosity appeared in the gas until the total potential difference between the filament and the anode was about 25 volts, when the central luminous column suddenly appeared, very brilliantly, reaching from near the anode right up to the grid. 'I he lower part of this luminosity was of a bright orange colour and the upper end, nearer the grid, was blood-red. On gradually reducing the potential difference across the tube the column became shorter and no longer reached to the grid, and when the total difference of potential between the filament and the anode had been reduced to about 21.5 volts, the glow occupied only about $5 \mathrm{~mm}$. in the centre of the tube and was all of a crimson colour showing only principal series lines of the neon spectrum. On reducing the maximum electron velocity still further, the glow became smaller in size, and finally disappeared when the potential difference was between $20^{\circ}$ volts and 21 volts. It must be remembered that at the relatively high pressure of this experiment the mean free path of an electron in the gas is small (considerably less than $1 \mathrm{~mm}$.), so that very few of the electrons after acquiring the lowest velocity with which they can collide inelastically with neon atoms, viz. 11.8 volte, will travel to the anode without loss of energy. The appearance of luminosity is thus delayed until the potential gradient between the grid and the anode is such that the electrons can acquire 20 volts velocity in a considerably shorter distance. The fact that most of the electrons make inelastic collisions in the gas before they get near to the anode also explains why the glow, when it does appear, does not extend right down to this electrode at this high pressure. 


\section{Prot. Horton and Miss Davies on Electron lelocities}

The extension of the glow right up to the grid when it appeared at 25 volts in this experiment, even though the velocity of the electron stream on renching the grid was not enough to produce ionization, confirms in a striking manner the view that luminosity is produced at the recombination of positive ions and electrons. The positive ions are driven upwards by the electric field between the grid and anode and become neutralized, by electrons from the filament, in positions nearer to the grid than those in which they are formed. As the potential difference between the grid and anode is reduced, the positive ions being now produced nearer the anode recombine with electrons before they get near the grid, and so the luminosity no longer reaches up to that electrode. Similar evidence was obtained from experiments at lower pressures. In all of these the potential difference between the filament and the grid was maintained at a value well below the minimum ionization velocity. The luminosity made its first appearance at the anode, and, as the total potential difference between the filament and the anode was increased, the lum nous column gradually became longer and ultimately reached the grid. In one instance the potential difference between the filament and the grid was only $t$ volts when a luminous column extendiny. right up to the grid was obtained. Thus the lumino ity at the grid could not be due to ionization, but must have resulted from the recombination of the positive ions with electrons from the filament.

\section{Discussion of Results and Conrlusion.}

The experiments described in this paper lead to the conclusion that there is no luminosity produced in neon as a result of the first type of ionization, shown in our earlier paper to begin at $16^{\circ} .7$ volts. Since it is generally accepted that when ionization and subsequent recombination oceur, a complete line spectrum corresponding to the particular ionization in question is produced, this absence of luminosity in the case of the 167 volts ionization in neon suggests that the corresponding spectrum may consist entirely of lines outside the visible region.

It has also been shown in the course of the paper that the lines contained in the spectrum of the glow when first produced, at 20 volts, are all of the $(1.5, s-m, p)$, or principal series, type according to Paschen's classification ; while the lines which do not appear until the ionizing velocity 22.8 volts has been reached, are all of the 
for Production of Luminosity in Atmospherie Neon. 937

$(2, p-m, s)$ and $(2, p-m, d)$, or subordinate series, types. On the Bohr-Sommerfeld theory, spectrum lines have their origin in the movements of an electron within an atom from one temporary position of stability (or temporary orbit) to another, in which the energy of the system is smaller. Thus the series of lines $(1 \cdot 5, s-m, p)$ result from the return of an electron to the $1 \cdot 5, s$ position of stability after having been displaced to one of the positions designated by $2 p, 3 p, 4 p$, \&c. It is generally assumed that when an atom is ionized and subsequent recombunation takes place, the electron, in returning to the orbit normally occupied, may stop temporarily in any of the intermediate stable orbits. In falling between any two temporary positions it causes the system to emit a radiation, the frequency of which corresponds to the energy differences between these two positions. Thus, when recombination takes place on a considerable scale, a spectrum consisting of lines corresponding to all possible displacements should result. The appearance of one set of lines at the ionization velocity 20 volts, and another set of lines at $22 \cdot 8$ volts, therefore indicates either that two entirely different elements are being ionized at these two velocities, or that displacements between quite different orbits in similar atoms are occurring. In order to distinguish between these two possibilities, it is necessary to ascertain whether there exists any close relationship between the constants of the particular subordinate series of which members appear when the highest ionization velocity is passed, and the constants of the particular principal series of which lines appear at 20 volts. A consideration of Table IV given below shows that there are instances in which lines of the series $(1 \cdot 5, s-m, p)$ appear at 20 volts, and lines of the corresponding $(2, p-m, s)$ series do not appear until the electron velocity reaches $22 \cdot 8$ rolts.

\section{TABLE IV.}

\begin{tabular}{|c|c|}
\hline \multicolumn{2}{|c|}{$\begin{array}{l}\text { Instances of lines of } \\
\text { series } 1.5, s-m, p \\
\text { appenring at } 20 \text { volts. }\end{array}$} \\
\hline$\lambda$. & Series. \\
\hline 507 & $15, s_{4}-2, p_{x}$ \\
\hline 678 & $1-5, s_{2}-2, p_{4}$ \\
\hline 6402 & $1 \cdot 5, s_{\tilde{0}}-2, p_{9}$ \\
\hline
\end{tabular}

\begin{tabular}{|c|c|}
\hline \multicolumn{2}{|c|}{$\begin{array}{l}\text { Instances of lires of the } \\
\text { corresponding } 2, p-m, s \text { ser } \\
\text { appearin at } 22 \cdot 8 \text { volts. }\end{array}$} \\
\hline$\Gamma_{\lambda}$. & Series. \\
\hline 5222 & $2, p_{y}-45, s_{4}$ \\
\hline 5298 & $2, p_{4}-4 \cdot 5, s_{2}$ \\
\hline 5189 & $2, p_{9}-1 \overline{0}, s_{3}$ \\
\hline
\end{tabular}

Each column of the table includes lines of the two types of series found by Paschen to occur in the neon spectrum, 


\section{Prof. Horton and Miss Davies on Electrun Velocities}

viz. those which follow the Ritz interpolation formula with great exactitude, without any modification of the combination principle, and those which require either a modification of this principle or a modification of the Ritz expression. In the paper already referred to, Paschen gave the extension of the Ritz interpolation formula, in the case of each series of the second type, which was necessary in order that the combination principle might hold. In a more recent paper * he has shown that if the combination principle is modified by the addition of a constant to the combination values of all terms of these series, the values of the terms so obtained can be expressed by formulæ of the exact Ritz type with greater accuracy than by the formulæ given earlier. The series to which $\lambda 6678$ and $\lambda 5298$ belong are of the type which requires this modification of the combination principle; but the series to which the other lines belong were satisfactorily expressed in the earlier paper. Thus both types of series are represented in the spectrum of the luminosity produced at 20.0 volts and in the additional lines which appear when the ionization velocity of $22 \cdot 8$ volts is passed.

The fact that the combination principle holds without any modification between some of the lines excited at $20 \cdot 0$ volts and some of those excited at $22 \cdot 8$ volts, rules out the possibility of these two ionization velocities corresponding to the ionization of two distinct elements. We are thus forced to the conclusion that the different ionization velocities which have been detected correspond to the removal of differently situated electrons from the neon atom, and hence that lines as closely related as those of a principal and the corresponding subordinate series arise from the return of electrons removed from different positions within the atom.

From the position of argon in the Periodic Table of the elements, its atom would be expected to have an arrangement of external electrons bearing some resemblance to that occurring in neon. We should therefore expect that argon would have more than one ionization velocity corresponding to the removal of a first electron from its atom, and that its spectrum could be excited in stages. We have not yot investigated the minimum electron velocities required for the excitation of spectrum lines in argon, but nur experiments on the ionization of this gas gave no indication of the existence of more than one critical electron velocity for the production of ionization, and nothing is recorded in the work of other investigators which suggests that

* F. Paschen, Ann. der Physik, vol. 1xiii. p. 201 (1920). 
for Production of Luminosity in Atmospheric Neon. 939

more than one ionization velocily has been detected. In employing the method which we used for the investigation of critical electron velocities in argon and other gases, the detection of a second or third critical velocity for the removal of a first electron from the atom, if more than one critical velocity existed, would depend upon the relative probabilities of ionization resulting from encounters between atoms and electrons having velocities in excess of the critical value, in each case. It is doubtiul whether the higher critical velocities would always be detected, unless the corresponding radiation velocities were intermediate to the ionization velocities.

Of the theories of atomic structure which have been put forward, that proposed by Lewis and by Langmuir * affords an explanation of the greatest number of different phenomena. This theory, which was originally evulved to account for the facts of chemical combination and valency, has since obtained considerable support from the recent work on X-rays and crystal structure $\dagger$, and has been found to be consistent with the results of the investigations on magnetism and atomic structure $\ddagger$. So far, however, it has not been made to supply any explanation of the excitation of spectrum lines and the series relations in spectra. In this direction the Bohr-Sommerfeld theory of the existence within the atom of a series of non-radiating orbits, any of which may be temporarily occupied by an electron, has been found the most satisfactory, but it has not yet adequately explained the existence of the soveral series of non-radiating orbits necessary to account for all the lines of the complicated spectra of elements whose atoms contain several electrons. The fact that in neon principal and corresponding subordinate series appear to be associated with the transitions of electrons differently situated in the normal atom, seems likely to be of importance in this counexion.

According to the Lewis-Langmuir theory, the electrons in the atom of neun exist in two concentric shells, the inner shell containing two electrons and the onter shell the remaining eight electrons. In this model the electrons are either stationary or execute small oscillations about mean positions. Since the two electrons in the inner shell are closer to the nucleus than the eight outer electrons, it seems reasonable to assume that a much larger quantum of energy

* I. Langmuir, Phys. Rev. vol. xiii. p. 300 (1919).

+ W. L. Bragg', Phil. Mag. vol. xl. p. 169 (1920).

† A. E. Oxley, Proc. Roy. Soc. A. vol. xcriii. p. 264 (1921). 
would be required to remove one of these two electrons than is required to remove one from the outer shell. If, therefore, we interpret our results on the basis of this theory, each of the three ionization velocities found in neon would correspond to the removal of one of the eight outer electrons. Our results therefore indicate that in the outer shell of the neon atom electrons occupy three dissimilar positions with regard to the nucleus and inner shell. This conclusion is in contraliction to the theory of Lewis and Langmuir in its present form, but it is possible that the essential features of the theory might be retained without necessitating absolute similarity of position among the electrons in any particular shell.

\section{Ether, Light, and Matter. By Sir Oliver Lodge*}

I the Octoher 1913 number of the Phil. Mag. vol. xxvi. 1 pp. 636-673, there is a remarkable though highly speculative paper by Professor Bruce McLaren (killed, alas! in the war) in which, among other things, he attempts to explain gravity by treating matter as a source or sink of ether. The flow of ether which is thus necessarily postulated is supposed to transfer momentum from the destroyed portion to the boundary of its destruction, and thus virtually to exert a stress on the surface of transition, notwithstanding that matter is unlike a foreign body immersed in a stream, but is a peculiarity of the ether itself. He seems to think that physicists will object to locomotion of the ether, as perturbing to the rays of light; but so long as motion is irrotational it can be shown that rays of light are not affected, though the waves are carried along and made excentric. In other words the path of a specified unit of luminous energy is not altered by any irrotational drift, whatever happens to the wave fronts; for in a moving ether the rays, i.e. the paths of energy, are not normal to the wave front, and nothing perceptible need happen. That nothing happens is conceded by the Principle of Relativity; though an explanation of why nothing perceptible bappens, and the idea of drift of wave fronts, are foreign to that theory.

That nothing happens on ordinary theory, at least to the first order of aberration magnitude, follows thus :- If the

* Communicated by the Author. 